REVISTA CIENTÍFICA RURAL

ISSN: 1413-8263 2525-6912
Revista Técnico-Científica

\title{
COMPOSTOS BIOATIVOS NAS FOLHAS DE GENÓTIPOS DE MARACUJAZEIROS
}

\author{
Diego Weber ${ }^{*}$, Jessica Fernanda Hoffmann², Caroline Farias Barreto', Nathalia de Avila Madruga1, \\ Rosane Lopes Crizel' ${ }^{1}$, Jair Costa Nachtigal ${ }^{3}$, Fabio Clasen Chaves ${ }^{1}$, Marcelo Barbosa Malgarim ${ }^{1}$ \\ ${ }^{*}$ Engenheiro Agrônomo, Doutor. Departamento de Fitotecnia, Fruticultura de Clima Temperado, Universidade Federal de \\ Pelotas, Pelotas, Brasil. E-mail: dieweb@gmail.com \\ 2 Tecnóloga em Alimentos, Doutora. Departamento de Ciência e Tecnologia Agroindustrial, Universidade Vale do Rio dos \\ Sinos, São Leopoldo, RS, Brasil; \\ ${ }^{3}$ Engenheiro Agrônomo, Doutor, Pesquisador, Embrapa Clima Temperado, Pelotas, RS, Brasil.
}

\begin{abstract}
RESUMO: Objetivou-se avaliar os compostos bioativos das folhas de genótipos de maracujazeiros tradicionalmente produzidos no Brasil. O experimento foi conduzido e avaliado entre 2015 e 2016. Os tratamentos foram estabelecidos de acordo com os genótipos: Catarina Roxo, Urussanga, BRS Rubi do Cerrado, Catarina e BRS Sol do Cerrado. As variáveis avaliadas foram: compostos fenólicos totais, teor de flavonoides e capacidade antioxidante (DPPH e ABTS). Os genótipos Catarina e BRS Rubi do Cerrado demonstram maiores teores de fenóis totais em relação aos demais genótipos, para esta variável os menores teores foram observados em folhas dos genótipos Urussanga e Catarina Roxo. O genótipo BRS Rubi do Cerrado foi destaque para a variável flavonoides, diferenciando-se estatisticamente dos demais, a menor média foi observada para o genótipo Catarina Roxo, não se diferenciando estatisticamente do BRS Sol do Cerrado. As folhas do genótipo Urussanga demonstraram maior capacidade antioxidante, tanto para o sequestro do radical DPPH quanto para o radical ABTS. Conclui-se que os genótipos avaliados, tradicionalmente produzidos no Brasil, demonstram compostos bioativos nas folhas, com potencial para exploração.
\end{abstract}

Palavras-chave: Passiflora, flavonoides, antioxidante, fenóis.

\section{BIOACTIVE COMPOUNDS IN THE LEAVES OF PASSION FRUIT GENOTYPES}

ABSTRACT: This study aimed to evaluate the bioactive compounds from the leaves of passion fruit genotypes traditionally produced in Brazil. The experiment was conducted and and evaluated between 2015 and 2016. Treatments were established according to the genotypes: Catarina Roxo, Urussanga, BRS Rubi do Cerrado, Catarina e BRS Sol do Cerrado. The variables analyzed were: total phenolics, flavonoid content and antioxidant activity (DPPH and ABTS). Genotypes Catarina and' BRS Rubi Cerrado show higher total phenolic content than the other genotypes, 
for this variable the lower levels were observed in leaves of genotypes Urussanga and Catarina Roxo. The genotype BRS Rubi Cerrado was especially the flavonoid variable, differing statistically from the other, the lowest average was observed for genotype Catarina Roxo, not differing statistically from BRS Sol Cerrado. The leaves of Urussanga genotype showed higher antioxidant activity, both for the kidnapping of $D P P H$ radical and for the ABTS radical. It is concluded that the genotypes traditionally produced in Brazil demonstrate bioactive compounds in the leaves, with the potential for exploitation.

Keywords: Passiflora, flavonoid, antioxidant, phenols.

\section{INTRODUÇÃO}

As espécies mais cultivadas no Brasil e no mundo são: maracujá-amarelo (Passiflora edulis $f$. flavicarpa), maracujá-roxo (Passiflora edulis) e maracujá-doce (Passiflora alata), porém, o maracujá-amarelo ou azedo representa quase a totalidade do volume comercializado mundialmente (KISHORE et al., 2011; PIRES et al., 2011).

O maracujá é um fruto cultivado em países de clima tropical e subtropical, pertencente à famíliapassifloraceaea, do gênero passiflora(COELHO et a., 2016). Entretanto, o maracujazeiro também pode ser cultivado em áreas de clima temerado, como o caso da região sul do Brail (WEBER et al., 2016). A expansão do cultivo tem despertado interesseseconômicos, mas também nos compostos bioativos dessa planta.

As Passifloras constituem uma enorme riqueza, tanto ao nível econômico, nutricional, bem como de recursos genéticos. As Passifloras possuem ampla quantidade de compostos bioativos que podem ser utilizadas para tratamentos de doenças ou distúrbios em humanos (MARLIÉREet al., 2008;ZERAIK et al. 2010; CLARO et al., 2018).

Os genótipos comerciais de maracujá apresentam elevados teores de compostos bioativos, destacando-se os compostos antioxidantes, os carotenóides e os compostos fenólicos (ZERAIKet al. 2010). Esses compostos podem ser encontrados na polpa, na casca e nas folhas do maracujazeiro, e podem ser influenciados por diversos fatores, dentre eles o genótipo, as condições edafoclimáticas, o sistema de cultivo e o processamento pós-colheita (FALEIROet al., 2015; MONTEALEGRE et al., 2006). A polpa e a casca do maracujá apresentam elevados teores de compostos bioativos que podem propiciar inúmeros benefícios à saúde humana (FALEIROet al., 2015). 
A utilização dos compostos bioativos, extraídos do suco, folhas e dos resíduos agroindustriais do maracujá, se justifica economicamente quando aplicados em áreas como farmacêutica, cosmética, química e alimentícia (ARVANITOYANNIS, 2008; OLIVEIRAet al., 2009).A maior parte dos fitoterápicos utilizados na medicina provém das folhas. Neste contexto, poucos trabalhos demonstram está espécie como potencial medicinal, ou com compostos bioativos que justificam tal utilização. No entanto, é necessário ampliar o conhecimento sobre os compostos existentes nas folhas de maracujazeiro.

Diante do exposto, o presente trabalho foi realizado com o objetivo de avaliar os compostos bioativos das folhas, compreendendo cinco genótipos comerciais de maracujazeiros de duas espécies de Passiflora.

\section{MATERIAL E MÉTODOS}

As folhas foram oriundas de maracujazeiros conduzidos no Centro Agropecuário da Palma (CAP), Faculdade de Agronomia Eliseu Maciel, Universidade Federal de Pelotas (UFPel), município do Capão do Leão (região de Pelotas), RS, Brasil, latitude 31052'00" S, longitude 52 $21^{\circ} 24^{\prime \prime}$ W GRW e 13 metros de altitude.

Foram utilizadas amostras oriundas de maracujazeiros-azedo (Passiflora edulis Sims) e maracujazeiro-doce (Passiflora alata Curtis). Os tratamentos consistiram: Seleção Passiflora edulis 'Catarina Catarina Roxo' (Epagri); Urussanga: Passiflora alata 'Urussanga' (Epagri); Passiflora edulis, BRS Rubi do Cerrado; Passiflora edulis 'Catarina' (Epagri); Passiflora edulis, BRS Sol do Cerrado.

Foram colhidos cinco quilos de folhas inteiras, no terço mediano dos ramos da planta para cada tratamento, em setembro de 2015. As folhas foram secas à sombra sob temperatura ambiente. Após, as folhas estarem totalmente secas, as amostras foram moídas em liquidificador industrial (cerca de $1 \mathrm{~mm}$ ) e armazenadas em temperatura ambiente em frascos de vidro protegido da luz, até o momento das análises.

Para análise de compostos fenólicos, flavonoides e atividade antioxidante foram pesados duas gramas de amostra triturada e adicionado $20 \mathrm{~mL}$ de metanol P.A. A mistura foi homogeneizada em ultra turrax (7500 rpm) por um minuto e submetido a centrifugação a $7000 \mathrm{rpm} / 15$ minutos $\left(15^{\circ} \mathrm{C}\right)$. O sobrenadante foi coletado em tubos falcon de $15 \mathrm{~mL}$ e armazenados em freezer a $-20^{\circ} \mathrm{C}$. 
Os compostos fenólicos totais foram determinados pelo método baseado na reação com o reagente Folin-Ciocalteau, adaptado de Singleton e Rossi, (1965). Para a reação, uma alíquota de $250 \mu \mathrm{L}$ do sobrenadante foi diluída em $4 \mathrm{~mL}$ de água ultrapura e o controle preparado com $250 \mu \mathrm{L}$ de metanol. Foram então adicionados $250 \mu \mathrm{L}$ de Folin-Ciocalteau 0,25N homogeneizando-se em vortex (Phoenix, AP-56), após 3 minutos de reação foi adicionado $500 \mu \mathrm{L}$ de carbonato de sódio $1 \mathrm{~N}$, após $2 \mathrm{~h}$ de reação à temperatura ambiente foi efetuada a leitura da absorbância em espectrofotômetro (Jenway, 6700) no comprimento de onda de $725 \mathrm{~nm}$. Os resultados foram expressos em mg equivalente de ácido gálico em $100 \mathrm{~g}$ de amostra em base úmida.

O teor de flavonóides totais foi determinado conforme a reação colorimétrica pelo método proposto por Zhishen et al. (1999) com adaptações. Adicionou-se 500 $\mu \mathrm{L}$ do extrato em tubo de falcon de $15 \mathrm{~mL}$ juntamente com $2 \mathrm{~mL}$ de água destilada e $150 \mu \mathrm{L}$ de $\mathrm{NaNO}_{2}$ (5\%), deixou-se reagir durante $5 \mathrm{~min}$., em seguida adicionou-se $150 \mu \mathrm{L}$ de $\mathrm{AlCl}_{3}$ (10\%), deixou-se reagir por mais 6 min., após adicionou-se $1 \mathrm{~mL}$ de $\mathrm{NaOH} 1 \mathrm{~mol} \mathrm{~L}^{-1}$ e 1,2 mL de água destilada, em seguida realizou-se a leitura em espectrofotômetro a $510 \mathrm{~nm}$. Os resultados foram expressos em $\mathrm{mg}$ de equivalente de catequina $100 \mathrm{~g}^{-1}$ de amostra, através de curva realizada com padrão de catequina.

A atividade antioxidante, utilizando o radical livre 2,2-difenil-1-picril-hidrazila (DPPH), foi determinada pelo método adaptado de Brand-Williams et al. (1995). Para a reação, foi utilizado $100 \mu \mathrm{L}$ do extrato e adicionado $3,9 \mathrm{~mL}$ de solução de DPPH em metanol. A solução foi então homogeneizada e os frascos mantidos no escuro por $24 \mathrm{~h}$, quando foi realizada a leitura no comprimento de onda de $517 \mathrm{~nm}$ em espectrofotômetro (Jenway, 6700). Os resultados foram expressos em porcentagem de inibição do radical DPPH.

O potencial antioxidante utilizando o radical 2,2-azinobis(3-etilbenzotiazolina6-ácido-sulfônico) (ABTS)foi determinado pelo método adaptado de Rufino et al. (2007). Foi primeiramente preparado o radical ABTS a partir da reação de $5 \mathrm{~mL}$ de solução de ABTS $(7 \mathrm{mM})$ com $88 \mu \mathrm{L}$ de uma solução de persulfato de potássio 140 mM. A mistura foi mantida no escuro, à temperatura ambiente, por 16 horas. Em seguida, dilui-se $1 \mathrm{~mL}$ desta mistura em álcool etílico até obter uma absorbância de $0,700 \mathrm{~nm} \pm 0,05 \mathrm{~nm}$ a $734 \mathrm{~nm}$. (reparada e usada apenas no dia da análise). 
Transferiu-se uma alíquota de $30 \mu \mathrm{L}$ do extrato (mesmo extrato obtido para a reação de fenóis totais) para tubos falcon com $3,0 \mathrm{~mL}$ do radical ABTS e homogeneizado em vortex (Phoenix, AP-56), a leitura foi realizada no comprimento de onda de 734 nm (Jenway, 6700) após 6 minutos de reação. Os resultados foram expressos em porcentagem de inibição do radical ABTS.

$O$ experimento foi realizado em delineamento inteiramente casualizado, com 3 repetições. Para as variáveis, procedeu-se a análise de variância pelo teste $\mathrm{F} \mathrm{e}$, quando o efeito de tratamento foi significativo, realizou-se teste de comparação de médias (Duncan) ao nível de $5 \%$ de probabilidade de erro. Os dados foram tabulados e interpretados pelo programa estatístico WinStat (MACHADO; CONCEIÇÃO, 2007).

\section{RESULTADOS}

As concentrações de compostos fenólicos totais nas folhas foram menores nos genótipos Urussanga e Catarina Roxo, em relação aos demais genótipos (Tabela 1). Os genótipos superiores em compostos fenólicos totais nas folhas foram BRS Rubi do Cerrado e Catarina.

Tabela 1: Compostos fenólicos totais e flavonoides em folhas de maracujazeiros de diferentes genótipos..

\begin{tabular}{ccc}
\hline Genótipo & Compostos fenólicos totais $^{1}$ & Flavonoides $^{2}$ \\
\hline BRS Rubi do Cerrado & $128,85 \pm 10,12 \mathrm{a}$ & $413,29 \pm 27,31 \mathrm{a}$ \\
Catarina & $139,35 \pm 5,59 \mathrm{a}$ & $337,44 \pm 20,56 \mathrm{~b}$ \\
Urussanga & $52,85 \pm 4,61 \mathrm{c}$ & $333,25 \pm 8,31 \mathrm{~b}$ \\
BRS Sol do Cerrado & $75,71 \pm 10,65 \mathrm{~b}$ & $304,75 \pm 62,33 \mathrm{bc}$ \\
& & $255,66 \pm 15,22 \mathrm{c}$
\end{tabular}

Letras iguais na coluna não diferem significativamente pelo teste Duncan $(p \leq 0,05) .{ }^{1} \mathrm{mg}$ ácido gálico equivalente $100 \mathrm{~g}^{-1}$ peso seco (ps); ${ }^{2} \mathrm{mg}$ catequina equivalente $100 \mathrm{~g}^{-1} \mathrm{ps}$;

Para o teor de flavonoides, o genótipo BRS Rubi do Cerrado demonstrou superioridade em relação aos demais (Tabela 1). O genótipo Catarina Roxo e o BRS 
Sol do Cerrado apresentaram valores de flavonoides inferiores aos demais genótipos.

A atividade antioxidante utilizando o radical DPPH foi superior para o genótipo Urussanga, seguido do genótipo BRS Rubi do Cerrado (Tabela 2). Enquanto os valores inferiores foram observados para os genótipos Catarina, BRS Sol do Cerrado e Catarina Roxo.

O genótipo Urussanga foi superior para atividade antioxidante (ABTS), diferenciando-se estatisticamente dos demais. Enquanto que a menor atividade antioxidante (ABTS) foi observada para o genótipo BRS Sol do Cerrado.

Tabela 2: Atividade antioxidante em folhas de maracujazeiros de diferentes genótipos.

\begin{tabular}{ccc}
\hline Genótipo & $\mathrm{DPPH}^{1}$ & $\mathrm{ABTS}^{1}$ \\
\hline BRS Rubi do Cerrado & $11,33 \pm 1,41 \mathrm{~b}$ & $12,43 \pm 0,12 \mathrm{~b}$ \\
Catarina & $6,33 \pm 0,15 \mathrm{c}$ & $9,96 \pm 0,37 \mathrm{c}$ \\
Urussanga & $18,83 \pm 1,65 \mathrm{a}$ & $17,99 \pm 0,25 \mathrm{a}$ \\
BRS Sol do Cerrado & $6,28 \pm 0,55 \mathrm{c}$ & $7,67 \pm 0,87 \mathrm{~d}$ \\
Catarina Roxo & $5,67 \pm 1,26 \mathrm{c}$ & $10,67 \pm 1,37 \mathrm{bc}$ \\
\hline
\end{tabular}

\section{DISCUSSÃO}

Neste estudo os valores de fenóis totais apresentou em média $91,54 \mathrm{mg}$ ácido gálico equivalente $100 \mathrm{~g}^{-1}$ peso seco, conforme a Erro! Fonte de referência não encontrada.. No entanto, Salles (2014) verificaram valores inferiores ao observado no presente estudo. Esses autores verificaram em média de 4,67 mg ácido gálico equivalente $100 \mathrm{~g}^{-1}$ peso seco presentes no extrato seco de Passiflora edulis, utilizando o método de Folin-Ciocalteau,

Rodrigues (2012) encontrou teores de fenóis totais entre 1000 a 5000 mg de ácido gálico equivalente $100 \mathrm{~g}^{-1}$ de peso seco, para a espécie Passiflora alata e para o genótipo BRS Sol do Cerrado. Valores acima dos encontrados neste experimento.

Com o objetivo de estabelecer um critério de valores para as quantidades de fenóis totais, Vasco et al. (2008) estabeleceram as seguintes categorias: baixos 
( $<1000 \mathrm{mg}$ de ácido gálico $\left.100 \mathrm{~g}^{-1}\right)$, médios $\left(1000-5000 \mathrm{mg}\right.$ de ácido gálico $\left.100 \mathrm{~g}^{-1}\right)$ e altos teores (>5000 $\mathrm{mg}$ de ácido gálico $100 \mathrm{~g}^{1}$ ) para o peso seco. Com isso, de acordo com os resultados deste trabalho, todos os genótipos avaliados demonstraram baixos teores de fenóis.

Neste estudo observou-se variação da concentração de compostos fenólicos de acordo com o genótipo estudo. Conforme Meyerset al. (2003), essas variações podem estar fortemente relacionadas com grau de maturação, sazonalidade, além do genótipo em questão. Estudos com maracujá também relataram variação no conteúdo fenólico em frutos de diferentes genótipos (Ramaiya et al. 2013; CasierraPosada e Jarma-Orozco, 2016). Esses autores também relatam que o local do cultivo pode influenciar na concentração dos compostos bioativos.

Os teores de flavonoides nas folhas apresentaram valores próximos entre os genótipos de maracujazeiros (Tabela 1). Pode ser observado no presente trabalho, que o genótipo Urussanga, representante da espécie Passiflora alata, demonstrou similaridade em relação aos genótipos da espécie Passiflora edulis, sendo apenas inferior ao genótipo BRS Rubi do Cerrado e superior ao genótipo Catarina Roxo. Segundo Chabariberiet al. (2008), avaliando flavonoides em diferentes espécies de Passifloras, encontraram teores entre as espécies de Passiflora edulis e Passiflora alata, muito próximos entre si.

Petryet al. (2001), avaliando os teores de flavonoides, através de análise espectroscópica por ultravioleta, do extrato hidroetanólico das folhas das espécies Passiflora edulis e Passiflora alata, observaram para Passiflora edulis maior conteúdo de flavonoides em relação aos extratos de Passiflora alata e Passiflora incarnata. Neste contexto, podemos relatar a superioridade do genótipo BRS Rubi do Cerrado (Passiflora edulis) em relação ao genótipo Urussanga (Passiflora alata) neste experimento.

O potencial antioxidante utilizando o radical ABTS foi muito semelhante ao comportamento do radical DPPH (Tabela 2). A atividade antioxidante utilizando o radical DPPH e ABTS foi superior para o genótipo Urussanga. Cazarin et al. (2014) encontraram em frutos de maracujá amarelo, a capacidade antioxidante variando de $46,4 \%$ a $29,6 \%$. No entanto, os valores encontrados nesse estudo são menores que os reportados por Cazarin et al. (2014), devido que nesse trabalho o material analisado foi as folhas. 
Da mesma forma como observado por Alves (2013), a capacidade das amostras em inibirem o radical ABTS foi muito semelhante ao do DPPH. No entanto, a percentagem de inibição do radical ABTS foi claramente superior à percentagem de inibição de radicais DPPH, mostrando que as amostras apresentam elevada capacidade bloqueadora de radicais $A B T S$. $O$ que não aconteceu neste experimento com folhas, considerando assim que a inibição dos radicais ABTS e DPPH foram muito semelhantes em termos de valores

\section{CONCLUSÕES}

O estudo comprovou a alta diversidade de compostos bioativos presentes nos genótipos de maracujazeiros, justificando seu potencial. O genótipo Urussanga demonstra maior potencial antioxidante. Os genótipos BRS Rubi do Cerrado e Catarina demonstram maior potencial para compostos fenólicos. O genótipo BRS Rubi do Cerrado demonstra maior potencial para flavonoides.

\section{AGRADECIMENTOS}

À Coordenação de Aperfeiçoamento de Pessoal de Nível Superior (Capes), pela concessão da bolsa; à Universidade Federal de Pelotas, Epagri, Embrapa Cerrados e Embrapa Clima Temperado pelo apoio e financiamento. 


\section{REFERÊNCIAS}

ALVES, A.I.P. Contributo para a caracterização química e capacidade antioxidante de diferentes partes de Passiflora edulis Sims edulis. 2013. $114 \mathrm{f}$. Dissertação. (Mestrado em Qualidade e Segurança Alimentar) - Escola Superior Agrária de Bragança, Bragança, 2013.

ARVANITOYANNIS, I.S.; VARZAKAS, T.H. Fruit/Fruit juice waste management: treatment methods and potential uses of treated waste. In: ARVANITOYANNIS, I. S. Waste Management for the food industries. Amsterdam: Academic Press, 2008. p.579-628,

BRAND-WILLIAMS, W., CUVELIER, M., BERSET, C. Use of a Free Radical Method to Evaluate Antioxidant Activity.Food Science and Technology, v.28, p.25-30, 1995.

CASIERRA-POSADA, F.; JARMA-OROZCO, A. Nutritional composition of Passiflora species. In: Simmonds, M. S. J.; Preedy V. R. (Eds.). Nutritional composition of fruit cultivars. London: Academic, 2016.

CAZARIN, C.B.B.; SILVA, J.K.; COLOMEU, T.C.; ZOLLNERI, R. de L.; MARÓSTICA JUNIOR, M.R. Antioxidant capacity and chemical composition of passion fruit peel (Passiflora edulis). Ciência Rural, v. 44, p.1699-1704, $2014 . \quad$ DOI: doi.org/10.1590/0103-8478cr20131437

CLARO, M. de L.; RODRIGUES, G.P.; TEIXEIRA, S.A. Propriedades funcionais da casca do maracujá amarelo (Passiflora edulis) na síndrome metabólica Functional properties of yellow passion fruit bark (Passiflora edulis) in metabolic syndrome. Demetra, v. 13, p. 181-194, $2018 . \quad$ DOI: 10.12957/demetra.2018.28957

CHABARIBERI, R. de A.O., POZZI, A.C.S., ZERAIK, M.L.; YARIWAKE, J.H. Determinação espectrométrica dos flavonóides das folhas de Maytenus (Celastraceae) e de Passiflora (Passifloraceae) e comparação com método CLAE- 
UV. Revista Brasileira de Farmacognosia, v.4, p. 860-864, 2009. DOI: http://dx.doi.org/10.1590/S0102-695X2009000600011

COELHO, E.M.; AZÊVEDO, L.C.; UMZA-GUEZ, M.A. Fruto do maracujá: importância econômica e industrial, produção, subprodutos e prospecção tecnológica. Cadernos de prospecção, v. 9, n. 3, p.323-336, 2016. DOI: dx.doi.org/10.9771/S.CPROSP.2016.009.037

FALEIRO, F.G.; JUNQUEIRA, N.T.V.; COSTA, A.M. Ações de pesquisa e desenvolvimento para o uso diversificado de espécies comerciais e silvestres de maracujá (Passiflora spp.). Documentos 329 Embrapa Cerrados, 128 Embrapa, p. 26, 2015.

KISHORE, K.; PATHAK, K.A.; SHUKLAR, R.; BHAR, R. Effect of storage temperature on physic-chemical and sensory attributes of purple passion fruit (Passiflora edulis Sims). Journal Food Scienci Technology, v. 48, p.484-488. 2011. DOI: http://dx.doi.org/10.1007/s13197-010-0189-8.

MACHADO, A.; CONCEIÇÃO, A.R. Programa estatístico WinStat Sistema de Análise Estatístico para Windows. Versão 2.0. Pelotas: UFPel, 2002.

MARLIÉRE, L.D.P.; RIBEIRO, A.Q.; BRANDÃO, M.G.L.; KLEIN, C.H.; ACURCIO, F.A. Utilização de fitoterápicos por idosos: resultados de um inquérito domiciliar em Belo Horizonte (MG), Brasil. Revista Brasileira de Farmacognosia, v. 18, p. 754760, 2008. DOI: http://dx.doi.org/10.1590/S0102-695X2008000500021

MEYERS, K.J.; WATKINS, C.B.; PRITTS, M.P.; LIU, R.H. Antioxidant and anti proliferative activities of Strawberries. Journal of Agricultural and Food Chemistry,, v.51, pp.6887-6892, 2003. DOI: http://dx.doi.org/10.1021/jf034506n

MONTEALEGRE, R.R.; PECES, R.R.; VOZMEDIANO, J.L.C.; GASCUEÑA, J.M.; ROMERO, E.G. Phenolic compounds in skins and seeds of ten grape Vitis vinifera varieties grown in a warm climate. Journal of Food Composition and Analysis,v.19, p.687-693, 2006.DOI:https://doi.org/10.1016/j.jfca.2005.05.003 
OLIVEIRA, A.C. VALENTIM, I.B.; GOULART, M.O.F.; SILVA, C.A.; BECHARA, E.J.H.; TREVISAN, M.T.S. Fontes vegetais naturais de antioxidantes. Química Nova, v.32, p.689-702, 2009.DOI:http://dx.doi.org/10.1590/S010040422009000300013

PETRY, R.D.; REGINATTO, F.deP.F. Comparative pharmacological study of hydro ethanol extracts of Passiflora alata and Passiflora edulis leaves. Phytotherapy Research, v.2, p.162-164, 2001.DOI: https://doi.org/10.1002/ptr.694

PIRES, M. M.; GOMES, A.D.A.S.; MIDLEJ, M.M.B.C.; SÃO JOSÉ, A.R.; ROSADO, P.L.; PASSOS, H.D.B. Caracterização do mercado de maracujá. In: PIRES, M. M.; SÃO JOSÉ, A.R.; CONCEIÇÃO, A. O. (Eds.) Maracujá: avanços tecnológicos e sustentabilidade. Ilhéus, Editus, 2011. p. 21- 67.

RAMAIYA, D.S.; BUJANG, J.S.; ZAKARIA, M.H.; KING, W.S. and SAHRIR, M.A.S. Sugars, ascorbic acid, total phenolic content and total antioxidant activity in passion fruit (Passiflora) cultivars. Journal of the Science of Food and Agriculture v. 93, p. 1198-1205, 2013.

RODRIGUES, J.S.Q. Infusões à base de folhas de Passifloras do cerrado: compostos fenólicos, capacidade antioxidante in vitro e perfil sensorial. 2012. 155p. Dissertação (Mestrado em Nutrição Humana). Universidade de Brasília, Brasília, 2012.

RUFINO, M.doS.M.; ALVES, R.E; BRITO, E.S. de; MORAIS, S.M.de; SAMPAIO, C.deG.; PÉREZ-JIMÉNEZ, SAURA-CALIXTO, J.; F.D. Metodologia Científica : Determinação da Atividade Antioxidante Total em Frutas pela Captura do Radical Livre ABTS. Comunicado Técnico, 128 Embrapa, p. 3-6, 2007.

SALLES, B.C.C. Teor de polifenóis e avaliação dos efeitos do extrato seco de folhas de Maracujá Azedo (Passiflora edulis Sims) sobre a glicação de colágeno e a atividade plaquetária em ratos diabéticos. - 2014. 65 f. Dissertação 
(Mestrado em Ciências Farmacêuticas) - Universidade Federal de Alfenas, Alfenas, 2014.

SINGLETON V.L., ROSSI J.A. Colorimetry of total phenolics with phosphomolybdicphosphotungstic acid reagents.American. Journal of Enology and Viticulture, v. 16, p. 144-158, 1965.

VASCO, C.; RUALES, J.; KAMAL-ELDIN, A. Total phenolic compounds and antioxidant capacities of major fruits from Ecuador.Food Chemistry, v.111, n.4, p.816-823, 2008. DOI: https://doi.org/0.1016/j.foodchem.2008.04.054

WEBER, D.; ELOY, J.; GIOVANAZ, M.A.; FACHINELLO, J.C.; NACHTIGAL, J.C. Densidade de plantio e produção do maracujazeiro-azedo no sul do Brasil. Revista $\begin{array}{llll}\text { Brasileira de } \quad \text { Fruticultura, v.38, } & \text { p.99-106, } & 2016 .\end{array}$ DOI:https://dx.doi.org/10.1590/0100-2945-283/14

ZERAIK, M.L; PEREIRA, C.A.M.; ZUIN, V.G.; YARIWAKE, J.H. Maracujá: um alimentofuncional? Revista Brasileira de Farmacognosia, v.20, p.459-471, 2010. DOI: http://dx.doi.org/10.1590/S0102-695X2010000300026.

ZHISHEN, J., MENGCHENG, T.; JIANMING, W. The determination of flavonoid contents in mulberry and their scavenging effects on superoxide radicals. Food Chemistry, v.64, p.555-559, 1999. DOI: DOI: http://dx.doi.org/10.1016/S03088146(98)00102-2 